\title{
Editorial
}

\section{Applications of Nanomaterials in Multifunctional Polymer Nanocomposites}

\author{
Bin Li, ${ }^{1}$ Nazanin Emami, ${ }^{2}$ Guan Gong, ${ }^{3}$ and Weidong Song ${ }^{4}$ \\ ${ }^{1}$ Department of Mechanical Engineering, Wichita State University, 1845 Fairmount Street, Wichita, KS 67260-0133, USA \\ ${ }^{2}$ Department of Engineering Science and Mathematics, Luleå University of Technology, 97187 Luleå, Sweden \\ ${ }^{3}$ Swerea SICOMP AB, P.O. Box 271, 94126 Piteå, Sweden \\ ${ }^{4}$ PD Materials, Boeing Commercial Airplanes, Seattle, WA 98124, USA
}

Correspondence should be addressed to Bin Li; bin.li@wichita.edu

Received 20 December 2015; Accepted 21 December 2015

Copyright (C) 2016 Bin Li et al. This is an open access article distributed under the Creative Commons Attribution License, which permits unrestricted use, distribution, and reproduction in any medium, provided the original work is properly cited.

Incorporating functional nanomaterials into polymers is an effective approach to achieving enhanced performances and creating new functionalities for the lightweight material applications. The resulting polymer nanocomposites benefit from advantages of both polymer matrix and nanoreinforcement phase. For the past few decades, polymer nanocomposite has become one of the most attractive research areas drawing magnificent attentions from both academia and industry. It is designed by taking advantage of specific characteristics of components, with intention for broad applications such as lightweight structures and energy and biomedical applications. Selected original research articles were compiled in this special issue reviewing some recent achievement on multifunctional polymer nanocomposites.

P. Gao and Y. Zhang synthesized zinc borate nanowhisker and used it to fabricate fire retardant polystyrene nanocomposites. Meanwhile, the nanowhisker also improved the surface quality (smoothness) and mechanical properties. A similar scenario was also reported by C. Yufei et al. in surface modified titanium dioxide/polyurethane modified epoxy. The improved interfacial interactions and dispersion because of the surfactant on $\mathrm{TiO}_{2}$ led to the enhancement of both properties.

Y. Fu et al. dispersed nanosilica in epoxy to strengthen the shape memory effects of polymer matrix. The resulting nanocomposites showed both improved triple shape memory effects and the shape fixity properties. Nanocomposite fiber composed of ultrahigh molecular weight polyethylene and surface modified nanosilica was fabricated by J. Yeh and coworkers. Processing procedures were optimized leading to enhanced mechanical properties which can be attributed to the good dispersion and surface modification of nanosilica.

M. A. Awad et al. reported antibacterial activities of nanosilver modified polystyrene nanocomposites with intended application for food packaging, which can be used against Gram-negative bacteria Escherichia coli, Klebsiella pneumoniae, and Salmonella and Gram-positive bacteria Staphylococcus aureus. M. G. Albu et al. prepared nanocomposite scaffolds with nanosilver for biomedical applications, which showed a clear antiseptic activity against Escherichia coli.

These original research articles focused on critical issues in polymer nanocomposites including nanoparticle resources and dispersion, polymer/nanoparticle interface, and processing of nanocomposites and their effects on the structural properties and functionalities. We hope to enrich our readers and researchers with the state-of-the-art research by compiling these articles in this special issue.

Bin $\mathrm{Li}$

Nazanin Emami Guan Gong Weidong Song 

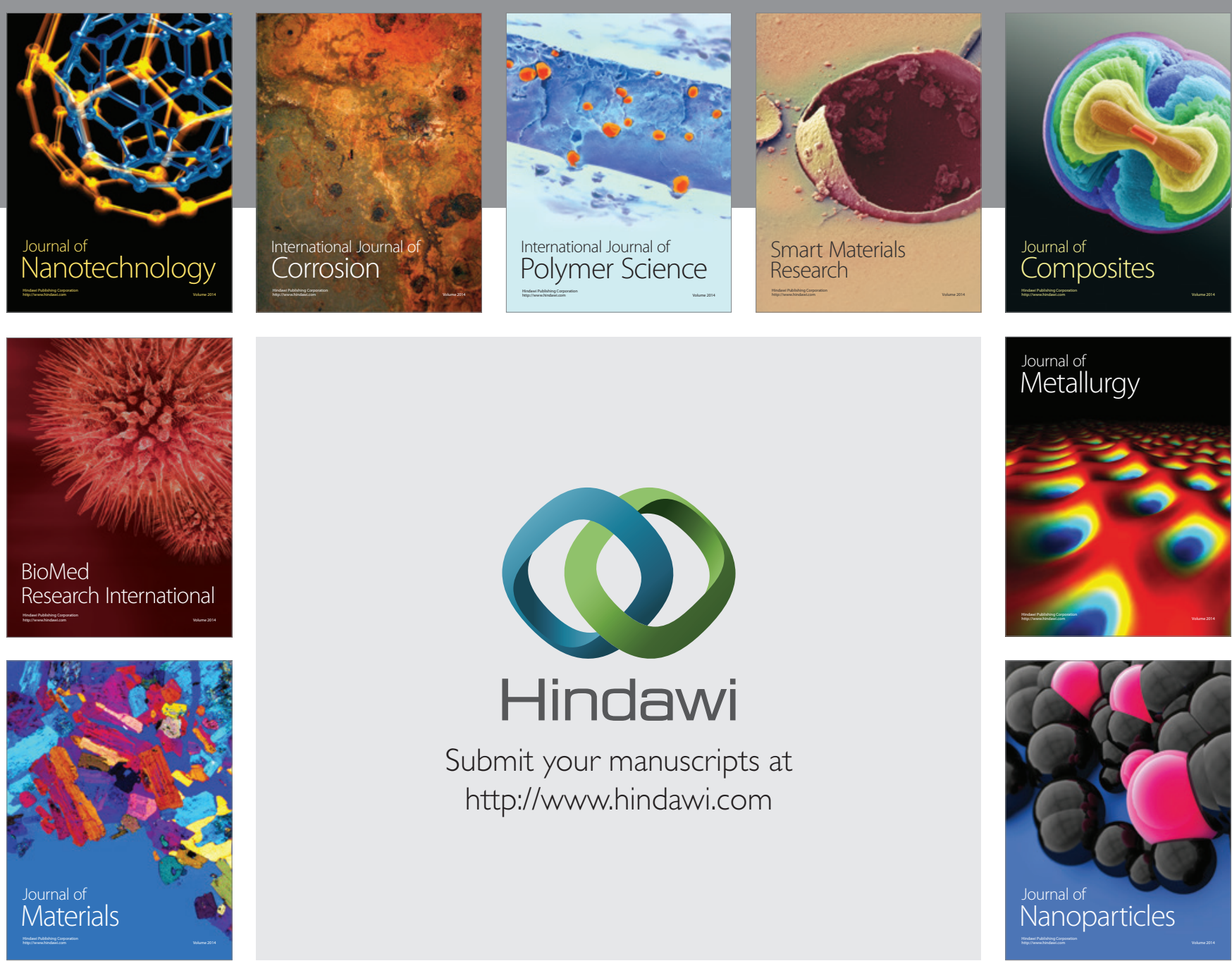

\section{Hindawi}

Submit your manuscripts at

http://www.hindawi.com

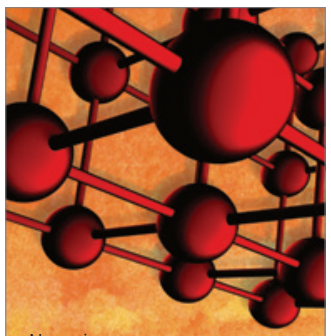

Materials Science and Engineering
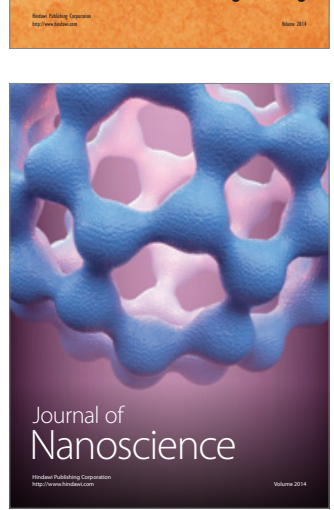
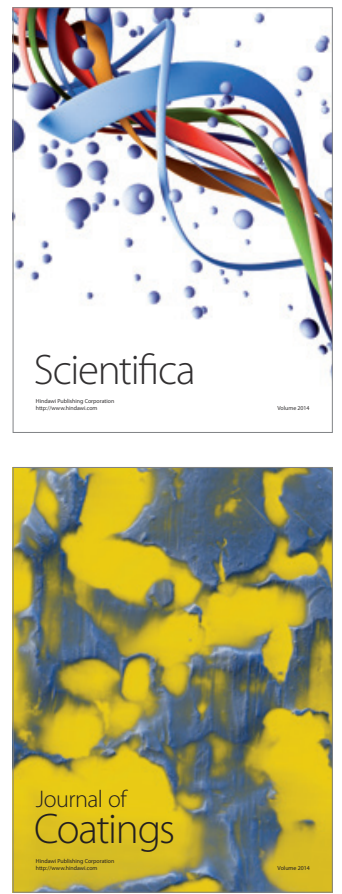
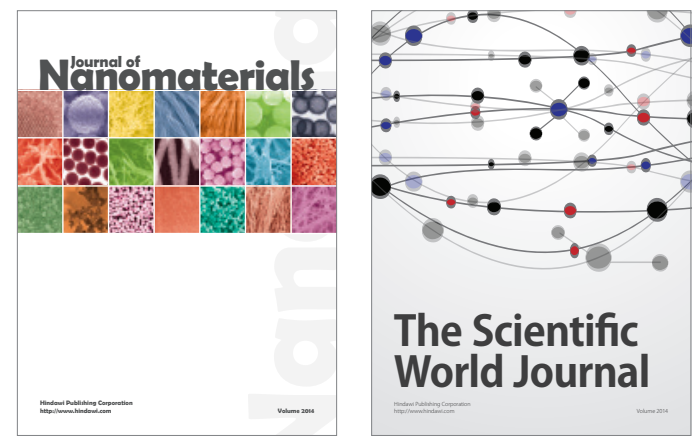

The Scientific World Journal
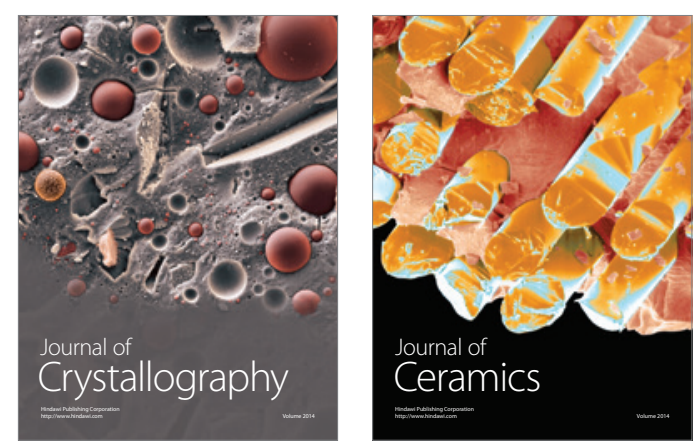
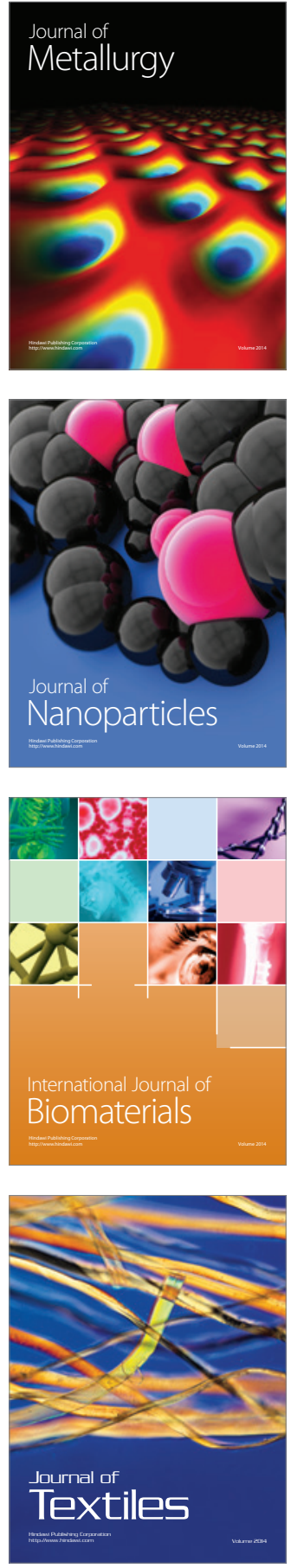